\title{
Clinical Profile and Outcome of Pediatric Acute Kidney Injury in Cameroon: Comparison between an Urban and a Semi-Urban Health Facility
}

\section{Fouda Menye Ebana Hermine Danielle ${ }^{1,2^{*}}$, Teuwafeu Denis George ${ }^{3,4}$, Halle Marie-Patrice ${ }^{2,5}$, Kaze Folefack Francois ${ }^{1,6}$, Ashuntantang Gloria ${ }^{1,7}$}

\author{
${ }^{1}$ Faculty of Medicine and Biomedical Sciences of Yaoundé, University of Yaoundé I, Yaoundé I, Cameroon \\ ${ }^{2}$ General Hospital of Douala, Douala, Cameroon \\ ${ }^{3}$ Faculty of Health Sciences, University of Buea, Buea, Cameroon \\ ${ }^{4}$ Regional Hospital of Buea, Buea, Cameroon \\ ${ }^{5}$ Faculty of Medicine and Pharmaceutic Sciences, University of Douala, Douala, Cameroon \\ ${ }^{6}$ Teaching Hospital of Yaoundé, Yaoundé, Cameroon \\ ${ }^{7}$ General Hospital of Yaoundé, Yaoundé, Cameroon \\ Email: ^mendjouf@yahoo.fr, dengeorgt@gmail.com,m_mahamat@yahoo.fr, tatangalex@yahoo.fr, patricehalle@yahoo.fr, \\ verlavincent@gmail.com,f_kaze@yahoo.fr,maglo09@hotmail.fr
}

How to cite this paper: Danielle, F.M.E.H., George, T.D., Marie-Patrice, H., Francois, K.F. and Gloria, A. (2018) Clinical Profile and Outcome of Pediatric Acute Kidney Injury in Cameroon: Comparison between an Urban and a Semi-Urban Health Facility. Open Journal of Nephrology, 8, 56-64. https://doi.org/10.4236/ojneph.2018.82007

Received: May 8, 2018

Accepted: June 26, 2018

Published: June 29, 2018

Copyright $\odot 2018$ by authors and Scientific Research Publishing Inc. This work is licensed under the Creative Commons Attribution International License (CC BY 4.0).

http://creativecommons.org/licenses/by/4.0/

c) (i) Open Access

\section{Abstract}

Introduction: Pediatric Acute Kidney Injury (AKI) seems to be a major cause of morbidity and mortality in Subsahara Africa. However, data on its epidemiology are scare and mainly originate from tertiary and urban health facilities such as large university Teaching hospitals with a nephrology service. Objective: The aim of this study was to compare the epidemiology of pediatric AKI in urban and semi-urban health facility. Patients and method: We conducted a retrospective study of 16 months in a tertiary urban hospital (General Hospital of Douala) and a secondary semi-urban health facility (Region hospital of Buea). Diagnostic of AKI was done using usual criteria. Age, sex distribution, etiologies of AKI, access to dialysis, renal recovery at hospital discharge, at 1 and 3 months and patient survival at hospital discharge was compared. Results: A total of 31 patients were included (GD 17 and RHB 14). Boys were more prevalent in the semi-urban setting ( $86 \%$ Vs $47 \% \mathrm{p}=0.029)$. Median age was comparable in the both group although most semi-rural patient were older (6.5 Vs 10 years $p=0.093)$. Hospital acquired AKI was only found in urban setting and account for $40 \%$ of pediatric urban AKI. Malaria related AKI (mainly black water fever) and sepsis were the main etiologies of AKI in urban and semi-urban milieu. Dialysis access was the same but $30 \%$ of 
children could not access to it in urban setting compare to none in semi-urban area. Reasons of non-access to dialysis were lack of pediatric material and financial constraints. In Hospital mortality was the same but was more severe amount patient who could not receive dialysis. Among the survivor, CKD was only found in urban setting. Conclusion: Although mainly due to malaria, pediatric AKI differs from urban tertiary and semi-urban secondary health facility in Cameroon. Hospital acquired AKI is only found in urban setting where children are younger and less boys. AKI in urban setting seems to be more severe with less access to dialysis and more risks of CKD.

\section{Keywords}

Pediatric AKI, Urban Tertiary Health Facility, Semi-Urban Secondary Health Facility, Malaria, Black Water Fever, Boys, Hospital Acquired AKI, CKD

\section{Introduction}

Acute kidney injury (AKI) is defined as an abrupt loss of kidney function that results in a decline in glomerular filtration rate, retention of nitrogenous waste products and dysregulation of extracellular volume and electrolytes. The term AKI has replaced acute renal failure, as it more clearly defines renal dysfunction as a continuum rather than a discrete finding of failed kidney function. Pediatric AKI is common and its epidemiology varies amount developed and resource-limited region. In high income region, pediatric AKI is typical hospital-acquired, affects critical ill children and is mainly secondary to other systemic illnesses or their treatment [1]. On the other hand, in undeveloped region, such as Sub-Sahara Africa, pediatric AKI is mainly community-acquired, affected young children and due to septicemia, malaria, severe dehydration and glomerular diseases [1] [2] [3]. In sub-Saharan Africa, although dialysis access rate in children had increase, mortality remains high, about $34 \%$, and increase to $70 \%$ if dialysis was indicated and not receive [2]. However, most of the studies available on epidemiology of AKI in this region have been conducted in tertiary and urban structure. In primary or secondary health facility, the picture of AKI can be worse since material and human resources are limited. Financial constraint and rural socio-cultural habit could also play a role. The aim of this study was to compare the epidemiology of pediatric AKI in urban and semi-urban health facility.

\section{Patients and Methods}

We conducted our study in 2 health facilities:

- The General Hospital of Douala (GHD) is located in the economic capital of country and serving the littoral region with a population estimated around 3 million. It is an urban tertiary institution. It has 320 beds, well-equipped laboratory, pediatric and radiology services. It serves also as teaching hospital 
of two Universities. It is the nephrology and oncology reference center of the Littoral region and the only public facility with RRT (Conventional hemodialysis) for AKI.

- The Buea regional hospital (BRH) is located in the capital of the South-West region and serves as the main reference hospital of this region with an estimated population around 1.5 million. It is a semi-urban secondary health facility. It has 180 beds, laboratory and radiology services as well as internal medicine and pediatric services. It is a teaching hospital of the faculty of Health Science of Buea. It is also the nephrology and HIV reference center of the region and hosts the unique hemodialysis center of this region.

No pediatric nephrologists are available in the both hospitals and children are managed in adult unit. Bicarbonate dialysate with polysulfone dialyzers are used and pediatric hemodialysis material is not routinely available. SYL project with peritoneal dialysis for children is more than $300 \mathrm{~km}$ away of the both facilities.

We retrospectively reviewed the medical records of all children admitted with the diagnostic of AKI from 1st November 2015 to 28th February 2017 at the GHD and the BRH. Children with known CKD and records with insufficient data for the diagnosis of AKI were excluded. We noted relevant clinical data including: etiology, mechanism and type of AKI, access to dialysis, in-hospital mortality and renal recovery at one and 3 months of diagnosis. The diagnostic of AKI was based on the following criteria:

- Increase or decrease in serum creatinine $>0.3 \mathrm{mg} / \mathrm{dl}$ from baseline within 48 hours.

- Increase or decrease in serum creatinine $>50 \%$ from baseline within 7 days.

- Urine output $<0.5 \mathrm{ml} / \mathrm{kg} /$ hour over 6 hours.

- Baseline creatinine referred to the first creatinine after the admission of the patient.

The KDIGO 2012 classification was used to stage the severity of AKI. Renal outcomes were reported only for survivors at hospital discharge and were evaluated at one and three months.

- Complete renal recovery was definite as normalization of serum creatinine.

- Partial recovery as persistence of renal failure without need of dialysis in those who were receiving dialysis or decrease of serum creatinine between 1.2 - 1.5 times baseline.

- No recovery as decrease of serum creatinine less than 1.2 times baseline or dependency on dialysis.

- Need of dialysis referred to patients with indications for dialysis.

- Access to dialysis to those with indications for dialysis and that were actually dialysed.

Diagnosis of etiologies, mechanism and type of AKI were clinical and the following definitions were used:

- Acute tubular necrosis was diagnosed based on history, presence of risk factors, urine indices when they were available and recovery with a polyuric phase. 
- Pre-renal AKI was diagnosed based on history, presence of risk factor, urea and creatinine ratio of more than 20 and urine indices when they were available.

- Nephrotoxins AKI was diagnosed based on a history of ingestion of know nephrotoxic drug (NSAI, aminoside...) or herbal remedies.

Analysis of the data was performed using SPSS version 20. Continuous data was summarized as mean or median as appropriate, while categorical data was presented as percentages. Logistic regression model was used to identify factors associated with poor renal outcome. The level of statistical significance was set at a p-value of $<0.05$. The study was approved by the GHD hospital ethic committee.

\section{Results}

A total of 31 patients were included; 17 at the GHD and 14 at the RHB. Boy were more prevalent in RHB (sex ratio 0.9 GHD and 3 RHB, $p=0.029$ ). Age distribution were similar in the both group although children in GHD were more young (median age $6 \mathrm{GHD}$ and $10 \mathrm{RHB}, \mathrm{p}=0.093$ ). Hospital acquired-AKI was only found in GHD (40\%). Acute tubular necrosis was the main type of AKI and black water fever was the main etiology in the both group. The entire patient at GHD had indication of dialysis but only 12 (70\%) access it. Reason of non-access were small weight $(n=3)$ and financial restraint $(n=2)$. In hospital mortality was the same (GHD 23\% and RHG 22\%). Death was associated with non-access to dialysis in GHD but not in RHB ( 4 of the five patients who could not access to dialysis in GHD died, $\mathrm{p}=0.002$ ). Among the survivor, all the semi-rural patient recovery while one patient $(10 \%)$ at GHD gone to chronic kidney disease (Figure 1, Table 1, Table 2, Table 3).

\section{Discussion}

The study compared the clinical profile of pediatric AKI between a tertiary urban hospital and a secondary semi-rural hospital. Hospital acquired AKI was only found in urban milieu and boy was more prevalent in semi-rural milieu. Malaria related AKI was the main AKI etiology and renal prognosis was more severe in urban setting.

We found a total of 17 and 14 children with AKI during the study period of 16 months in the GHD and the RHB respectively. These suggested a prevalence of pediatric AKI of 17 cases per year in the GHD and 10 cases per years in the RHB. Both a comparable to the 8.3 and 13.7 case per year found by Keita et al. in Senegal [4] and Olowu et al. in Nigeria respectively [5].

We found a difference in sex distribution between urban and semi-urban setting. Boys were more prevalent in semi-urban setting with a male-female ratio of 6:1 compared to $0.9: 1$ in urban milieu $(\mathrm{p}=0.029)$. Male predominance is usually found in AKI adult and children patients in sub-Saharan Africa [2]-[7]. This may reflect the bias in access to healthcare seen in these areas. Males who are usually 


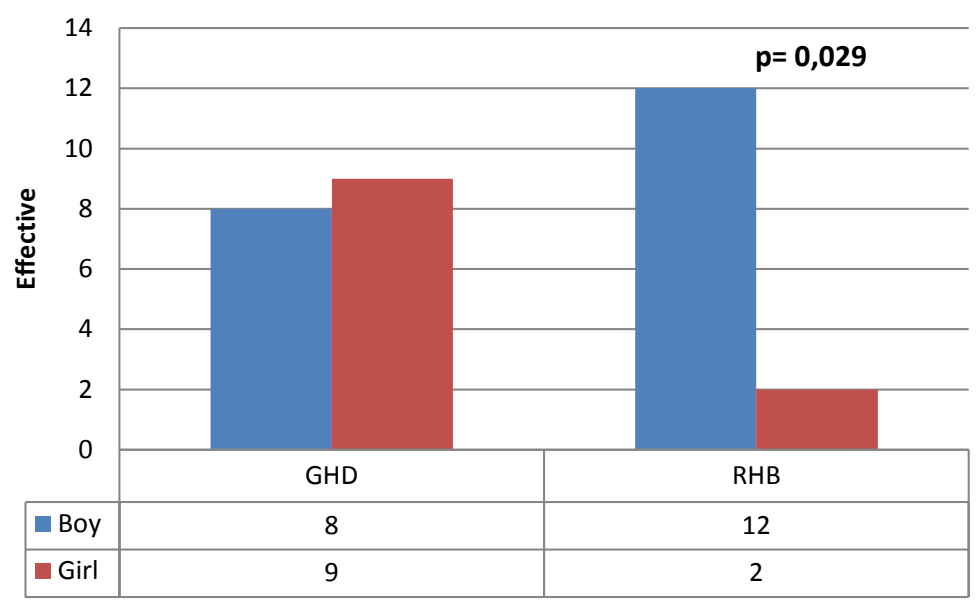

Figure 1. Sex distribution.

Table 1. AKI characteristics.

\begin{tabular}{|c|c|c|c|}
\hline & GHD (\%) $n=17$ & RHB (\%) $\mathrm{n}=14$ & $\mathrm{P}$ \\
\hline Age & & & 0.093 \\
\hline$\leq 5$ years & $8(47)$ & $3(21.5)$ & \\
\hline $5-10$ years & $4(23.5)$ & $4(28.5)$ & \\
\hline$>10$ years & $5(29.5)$ & $7(50)$ & \\
\hline AKI type & & & 0.009 \\
\hline Community-acquired & $10(60)$ & $14(100)$ & \\
\hline Hospital acquired & $7(40)$ & & \\
\hline Severity & & & 0.273 \\
\hline Stage 1 & 0 & $1(7)$ & \\
\hline Stage 2 & 0 & $1(7)$ & \\
\hline Stage 3 & $17(100)$ & $12(86)$ & \\
\hline AKI mechanism & & & 0.196 \\
\hline Pre-renal & 0 & $1(7)$ & \\
\hline Renal & $17(100)$ & $13(93)$ & \\
\hline ATN & $15(88)$ & $11(81)$ & \\
\hline HUS & $2(12)$ & - & \\
\hline AGN/NS & - & $2(14)$ & \\
\hline Etiologies & & & 0.551 \\
\hline Malaria & $8(47)$ & $7(50)$ & \\
\hline Black water fever & $6(36)$ & $4(22)$ & \\
\hline Sepsis & $4(23)$ & $4(22)$ & \\
\hline Gastroenteritis & $1(6)$ & $1(7)$ & \\
\hline Herbal plant & $2(12)$ & - & \\
\hline Renal disease & $2(12)$ & $2(14)$ & \\
\hline
\end{tabular}

*Renal disease included 2 Hemolytic and uremic syndrome (HUS), 1 Acute glomerulonephritis (AGN) and 1 nephrotic syndrom (NS). ATN acute tubular necrosis, GHD general hospital of Douala, RHB regional hospital of Buea 
Table 2. Dialysis indication and access.

\begin{tabular}{cccc}
\hline & GHD (\%) $\mathrm{n}=17$ & RHB (\%) $\mathrm{n}=14$ & $\mathrm{P}$ \\
\hline Dialysis indication & $17(100)$ & $12(86)$ & 0.07 \\
Dialysis access & 12 & 12 & 0.077 \\
Dialysis access ratio & 0.7 & 1 & \\
Reason of non-access to dialysis & & \\
Inadequate material & 3 & - & \\
Financial restraint & 2 & - &
\end{tabular}

Table 3. Outcomes.

\begin{tabular}{cccc}
\hline & GHD $(\%) \mathrm{n}=17$ & RHB $(\%) \mathrm{n}=14$ & $\mathrm{P}$ \\
\hline In hospital mortality & $4(23.5)$ & $3(21.5)$ & 0.61 \\
& Evolution among survivor & \\
Month 1 & & 0.045 \\
Complete recovery & $5(38.5)$ & $9(82)$ & \\
Partial recovery & $5(38.5)$ & - & - \\
Lost & $3(23)$ & $2(18)$ & \\
Month 2 & & - & \\
Complete recovery & 4 & - & \\
Chronic kidney disease & $1(10)$ & & \\
\hline
\end{tabular}

${ }^{*} \mathrm{n}=13 \mathrm{GHD}$ and $\mathrm{n}=11$ RHB.

the breadwinners access hospitals more frequently and; even in children, males are more considered than girls and therefore are more likely to be taken to hospital [8]. The difference between urban and semi-urban in our study probably reflects strongest socio-cultural habits in the semi-urban milieu.

Median age was comparable in the both group (GHD 6 years \# RHB 10 years $\mathrm{p}=0.093$ ). However, most of semi-urban children were more than 10 years and most of the urban children were less than 5 years. Abdelraheem et al. noted that neonates (27.1\%), Children between 1 - 5 years (19.3\%) and children of more than 10 years were mainly affected by AKI in Soudan [9]. Aloni in Congolese children found a median age of 6.7 years similar to the 6.9 years found by Keita et al. in Senegal; while in Nigeria, Esezobor found a median age of 4.8 years.

Hospital acquired AKI was only found in tertiary urban facility with a frequency of $40 \%$. In Nigeria, Esezobor reported a proportion of hospital acquired AKI of $17.1 \%$ and Olowu et al. of $27.2 \%$ [10]. Although our hospital acquired AKI frequency are greater, both Nigeria studies was also done in large tertiary teaching hospital. In Subsahara Africa, there are almost no data on the incidence of AKI in rural and semi-rural areas; making the magnitude and outcome of community-acquired AKI in Africa virtually unknown [1]. The fact that all the 
children of the semi-urban setting has community acquired AKI underline the need of study in such area.

In the both area, etiologies of pediatric AKI was the same and mainly due to malaria and bacterial infections. Malaria related AKI (MAKI) was the main cause of AKI in the both area and was mainly due to black water fever. Plasmodium falciparum was noted in all the case. AKI is found in $1 \%-4 \%$ of patient with P. falciparum infection and incidence can increase to $60 \%$ in severe malaria [11] [12] [13]. Need of dialysis is frequent (50\% - 80\%) in MAKI [14] as noted in our series (100\% in GHD and $86 \%$ in RHB). Patient with high parasitemia, children under 5 years, pregnant women, HIV patient are risk population [15]. Mortality of MAKI vary between $15 \%$ - 50\% [16] but early antimalarial treatment and dialysis is associated with improved survival and recovery of renal function [16] [17]. The overall mortality rate associated with MAKI was $20 \%$ (2/8 and $1 / 7$ patients respectively GHD and RHB) and was due to non-access to dialysis (GHD) and neurological complication (RHB). Black water fever was the main clinical form of MAKI in our cohort and the main form of pediatric AKI in urban and semi-urban area (GHD 35.3\% and RHB 33.4\%) as previously noted by Aloni in Democratic Republic of Congo [7]. It is a particular form P. falciparum malaria, characterized by acute intravascular hemolysis after administration of quinine or some other anti-malaria treatment (e.g. amodiaquine, mefloquine). It can be associated to glucose- 6 phosphate dehydrogenase deficit (which is highly found in black African) or not. AKI is frequent, result from hemolysis and renal histology shown acute tubular necrosis [18]. Passage of dark urine followed by oliguria is the typical presentation. Boni and al reported a mortality rate of 20.5\% among Congolese children with black water fever in Kinshasa [19]. However, in our series no death was associated with black water fever condition.

Renal disease such as hemolytic and uremic syndrome or acute glomerulonephritis was less frequent account for $12 \%$ and $14 \%$ of AKI in GHD and RHB respectively. In Nigeria, Esezobor found that primary kidney disease (mainly acute glomerulonephritis and nephrotic syndrome) was the main cause of pediatric AKI in Lagos Teaching Hospital, follow by sepsis and malaria [6].

AKI in urban tertiary facility was more severe since all the children need dialysis (100 \# $86 \mathrm{p}=0.07)$. Access to dialysis was comparable between the both groups although $30 \%$ of children in GHR could not benefit from dialysis compared to none in RHB. Reason of non-access to dialysis included lack of pediatric material [3] and financial constraints [2]. Mortality rate was high among patient who could not access to dialysis $(80 \% \# 18.2 \% \mathrm{p}=0.016)$. Olowu et al. also found high mortality rate $(70 \% \# 30 \% \mathrm{p}<0.0001)$ in children who did not receive a least one dialysis session [2].

In-hospital mortality rate was the same in urban and semi-urban milieu (23.5 \# $21.5 \mathrm{p}=0.61$ respectively). Among survivor, renal recovery was noted in all semi-urban children with available data while 1 CKD (10\%) was noted in GHD in a child with atypical hemolytic and uremic syndrom. Keita et al. in Senegal 
and Abdelraheen in Soudan found that $8 \%$ and $10 \%$ of their patients respectively went into CKD (4.9).

\section{Conclusion}

Clinical profiles of pediatric AKI in semi-urban secondary and tertiary urban health facility are different. Male predominance and older children are more prevalent in semi-urban area while female predominance and children $<5$ years are more concerned in urban milieu. Hospital acquired is only seen in urban tertiary setting and AKI in this setting seems to be more severe with $10 \%$ went into CKD. However etiologies are the same and mainly involved malaria related AKI.

\section{Limitation}

Since we conducted a retrospective study, our study had bias and limitation associated with this type of study. Another limitation is that no kidney biopsy was done and all the renal diagnostic was clinical. It could have also been interesting to have a third group of patient coming from a rural setting without a nephrology unit.

\section{References}

[1] Lameire, N., Van Biesen, W. and Vanholder, R. (2017) Epidemiology of Acute Kidney Injury in Children Worldwide, Including Developing Countries. Pediatric Nephrology, 32, 1301-1314. https://doi.org/10.1007/s00467-016-3433-2

[2] Olowu, W.A., Niang, A., Osafo, C., Ashuntantang, G., Arogundade, F.A., Porter, J., Naicker, S. and Luyckx, V.A. (2016) Outcomes of Acute Kidney Injury in Children and Adults in Sub-Saharan Africa: A Systematic Review. Lancet Glob Health, 4, e242-e250. https://doi.org/10.1016/S2214-109X(15)00322-8

[3] Halle, M.P., Lapsap, C.T., Barla, E., Fouda, H., Djantio, H., Moudze, B.K., Akazong, C.A. and Priso, E.B. (2017) Epidemiology and Outcomes of Children with Renal Failure in the Pediatric Ward of a Tertiary Hospital in Cameroon. BMC Pediatrics, 17, 202. https://doi.org/10.1186/s12887-017-0955-0

[4] Younoussa Kéita, E.F., Ka, M.M., Cissé, A., Sylla, M.M.M., Leye, A.T., Lemrabott, C., Dial, A.A., Ndongo, A., Ba, A., Niang, C., Moreira, B., Diouf and Sall, M.G. (2015) Acute Renal Failure (ARF) In Childen Diagnostic, Therapeutic, Evolutionary Aspecys and Prognostic about 50 Cases Diagnoses in a Pediatric Ward in Dakar. the review revue du Cames, 3, 55-60.

[5] Olowu, W.A. and Adelusola, K.A. (2004) Pediatric Acute Renal Failure in Southwestern Nigeria. Kidney International, 66, 1541-1548.

https://doi.org/10.1111/j.1523-1755.2004.00918.x

[6] Esezobor, C.I., Ladapo, T.A., Osinaike, B. and Lesi, F.E.A. (2012) Paediatric Acute Kidney Injury in a Tertiary Hospital in Nigeria: Prevalence, Causes and Mortality Rate. PLoS ONE, 7, e51229. https://doi.org/10.1371/journal.pone.0051229

[7] Aloni, M.N., Nsibu, C.N., Meeko-Mimaniye, M., Ekulu, P.M. and Bodi, J.M. (2012) Acute Renal Failure in Congolese Children: A Tertiary Institution Experience. Acta Paediatrica, 101, e514-e518. https://doi.org/10.1111/j.1651-2227.2012.02827.x

[8] Onah, M. and Govender, V. (2014) Out-of-Pocket Payments, Health Care Access and Utilisation in South-Eastern Nigeria: A Gender Perspective. PLOS ONE, 9, e93887. https://doi.org/10.1371/journal.pone.0093887 
[9] Abdelraheem, M., Ali, E.-T., Osman, R., Ellidir, R., Bushara, A., Hussein, R., Elgailany, S., Bakhit, Y., Karrar, M., Watson, A. and Abu-Aisha, H. (2014) Outcome of Acute Kidney Injury in Sudanese children-An Experience from a Sub-Saharan African Unit. Peritoneal Dialysis International, 34, 526-533. https://doi.org/10.3747/pdi.2013.00082

[10] Olowu, W.A., Adefehinti, O. and Bisiriyu, A.L. (2012) Hospital-Acquired Acute Kidney Injury in Critically Ill Children and Adolescents. Saudi Journal of Kidney Diseases and Transplantation, 23, 68-77.

[11] Randeree, I.G., Czarnocki, A., Moodley, J., Seedat, Y.K. and Naiker, I.P. (1995) Acute Renal Failure in Pregnancy in South Africa. Renal Failure, 17, 147-153. https://doi.org/10.3109/08860229509026251

[12] Naqvi, R., Ahmad, E., Akhtar, F., Naqvi, A. and Rizvi, A. (2003) Outcome in Severe Acute Renal Failure Associated with Malaria. Nephrology Dialysis Transplantation, 18, 1820-1823. https://doi.org/10.1093/ndt/gfg260

[13] Mehta, K.S., Halankar, A.R., Makwana, P.D., Torane, P.P., Satija, P.S., et al. (2001) Severe Acute Renal Failure in Malaria. Journal of Postgraduate Medicine, 47, 24-26.

[14] Barsoum, R.S. (2000) Malarial Acute Renal Failure. Journal of the American Society of Nephrology, 11, 2147-2154.

[15] Mishra, S.K. and Das, B.S. (2008) Malaria and Acute Kidney Injury. Seminars in Nephrology, 28, 395-408. https://doi.org/10.1016/j.semnephrol.2008.04.007

[16] Koh, K.H., Chew, P.H. and Kiyu, A. (2004) A Retrospective Study of Malaria Infections in an Intensive Care Unit of a General Hospital in Malaysia. Singapore Medical Journal, 45, 28-36.

[17] Zinna, S., Vathsala, A. and Woo, K.T. (1999) A Case Series of Falciparum Malariainduced Acute Renal Failure. ANNALS Academy of Medicine Singapore, 28, 578-582.

[18] Cerda, J., Bagga, A., Vijay, K. and Chakravarthi, R.M. (2008) The Contrasting Characteristics of Acute Kidney Injury in Developed and Developing Countries. Nature Clinical Practice Nephrology, 4, 138-153. https://doi.org/10.1038/ncpneph0722

[19] Bodi, J.M., Nsibu, C.N., Aloni, M.N., Lukute, G.N., Kunuanuna, T.S., Tshibassu, P.M. and Pakasa, N. (2014) Black Water Fever Associated with Acute Renal Failure among Congolese Children in Kinshasa. Saudi Journal of Kidney Diseases and Transplantation, 25, 1352-1358. https://doi.org/10.4103/1319-2442.144326 